Recepción: 05 / 04 / 2018

Aceptación: 19 / 05 / 2018

Publicación: 02 / 07 / 2018

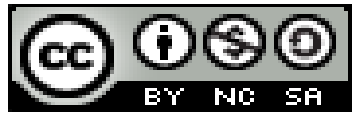

Ciencias de la educación

\title{
Retos de la Educación Superior en América Latina
}

\section{Challenges of higher education in Latin America}

\section{Desafios do ensino superior na América Latina}

\author{
Ana C. Bedoya-Gutiérrez ${ }^{\mathrm{I}}$ \\ ana-bedoya@hotmail.com
}

Correspondencia: ana-bedoya@hotmail.com

\footnotetext{
I Magister en Docencia Mención Gestión en Desarrollo del Currículo, Licenciada en Ciencias de la Educación Especialización Docencia Especial, Docente de la Universidad Técnica Luis Vargas Torres de Esmeraldas, Esmeraldas, Ecuador.
} 


\title{
Resumen
}

Se presenta un estudio de carácter bibliográfico e histórico de la vinculación de las transformaciones universitarias, en concordancia con los procesos históricos de modernización de las sociedades latinoamericanas, partiendo de la experiencia de la Reforma de Córdova en Argentina hace cerca de cien años, con especial énfasis en sus impactos en la transformación de la universidad latinoamericana, reconociendo en la Autonomía Universitaria uno de los principales logros del referido a la autonomía universitaria. Cada proceso de cambios y transformaciones de nuestras sociedades ha planteado nuevos desafíos a la educación superior, encarnada está en sus Universidades. Se revisan y contextualizan los cambios en las sociedades latinoamericanas en las consecutivas décadas, destacando los ocurridos en los años sesenta por el influjo revolucionario cubano, así como de los movimientos estudiantiles del mayo francés entre otros que habrían impactado en la efervescencia del movimiento estudiantil e incluso en el ámbito de las teorías sociales y la filosofía., para luego sufrir la reacción dictatorial militarista de los años 70, que derivaría a partir de los 80 en el ciclo neoliberal. El siglo XXI con sus complejidades y surgimiento de alternativas anti neoliberales, en el contexto de la sociedad del conocimiento, plantea retos que pueden orientarse de acuerdo a la mirada neoliberal o la socialista.

Palabras clave: retos; educación superior; América latina; siglo XXI.

\begin{abstract}
A bibliographic and historical study of the linking of university transformations is presented, in accordance with the historical processes of modernization of Latin American societies, based on the experience of the Reforma de Córdova in Argentina about one hundred years ago, with special emphasis in its impact on the transformation of the Latin American university, recognizing in University Autonomy one of the main achievements of the referred to university autonomy. Each process of changes and transformations of our societies has posed new challenges to higher education, embodied in its Universities. The changes in Latin American societies are revised and contextualized in the consecutive decades, highlighting those that took place in the 1960s due to the Cuban revolutionary influence, as well as the student movements of the French May among others that would have impacted the effervescence of the student movement and even in the field of social theories and philosophy, to then suffer the military
\end{abstract}


dictatorial reaction of the $70 \mathrm{~s}$, which would derive from the $80 \mathrm{~s}$ in the neoliberal cycle. The $21 \mathrm{st}$ century with its complexities and emergence of anti-neoliberal alternatives, in the context of the knowledge society, poses challenges that can be oriented according to the neoliberal or socialist view.

Keywords: Challenges; higher education; Latin America; XXI century.

\section{Resumo}

Um estudo da literatura e caráter histórico de ligar transformações universitários, de acordo com os processos históricos de modernização das sociedades latino-americanas, com base na experiência da Reforma de Córdoba, na Argentina cerca de cem anos atrás, com especial ênfase aparece em seu impacto sobre a transformação da universidade latino-americana, reconhecendo na Autonomia Universitária uma das principais conquistas da referida autonomia universitária. Cada processo de mudanças e transformações de nossas sociedades colocou novos desafios ao ensino superior, corporificados em suas Universidades. Nós revisamos e contextualizar as mudanças nas sociedades latino-americanas em décadas consecutivas, com destaque para as que ocorreram nos anos sessenta pelos cubanos influência revolucionária e estudantes circulação em França em Maio entre outros que têm impactado a efervescência do movimento estudantil e até mesmo no campo das teorias sociais e da filosofia, para então sofrer a reação ditatorial militar dos anos 70, que derivaria dos anos 80 no ciclo neoliberal. O século XXI, com suas complexidades e surgimento de alternativas anti-neoliberais, no contexto da sociedade do conhecimento, coloca desafios que podem ser orientados de acordo com a visão neoliberal ou socialista.

Palavras chave: Desafios; educação superior; América Latina; século XXI.

\section{Introducción}

A cien años de la Reforma de Córdova le toca a la universidad latinoamericana realizar un balance de los avances alcanzados por la educación superior en el sub continente y trazarse las metas a ser alcanzadas en el corto, mediano y largo plazo.

Córdova significó la superación de los anacronismos propios del viejo régimen universitario, caracterizado en el propio Manifiesto de Córdova de junio de 1998, por estar fundado en derechos de origen divino otorgados al profesorado universitario de entonces. Denunciaban la 
mediocridad que se había entronizado en las universidades de entonces, a las que acusaban de impulsoras de la insensibilidad y de ser reflejo de la decadencia social de esos tiempos, así como el autoritarismo retardatario expresado en la forma de escoger a las autoridades universitarias, y en el propio y decadente "principio de autoridad" ajeno a la propia naturaleza de enseñanza de la institución universitaria. "Se levantó contra un régimen administrativo, contra un método docente, contra un concepto de autoridad” (Manifiesto de Córdova, 1918).

Este movimiento reformista universitario y juvenil se desarrolló en un contexto de modernización continental, en el marco de la expansión del capitalismo a partir de la hegemonía inglesa y la transición del predominio de la sociedad tradicional a la sociedad moderna e industrial, de la cual habría sido argentina de las primeras en desarrollar. González (2011) refiere como antecedentes las guerras civiles intestinas que sufrió la argentina en la segunda mitad del siglo XIX, los procesos de inserción de la economía de ese país en la trama del capitalismo, bajo la égida de las oligarquías terratenientes asociadas con el capital británico, quienes habrían impulsado los cambios urbanos e industriales, pero manteniendo un régimen político extremadamente conservador.

Los logros de transformación universitaria de la Reforma de Córdova son reconocidos de manera generalizada. Citando a Tünnermann, López (2008), se atreve a afirmar que la Reforma de Córdova resultó ser "el más temprano enfrentamiento contra la universidad latinoamericana de viejo régimen marcando la entrada de Latinoamérica al siglo XX (López, 2008, p. 338), el cual se vería reflejado en una docena de reformas, a saber:

a) Autonomía Universitaria en sus aspectos políticos, docente, administrativo y económico, b) Elección de los cuerpos directivos, de las autoridades y de los organismos de co-gobierno por la comunidad universitaria, c) Concursos de oposición para la selección del profesorado, d) Libertad de cátedra, e) Gratuidad de la enseñanza, f) Reorganización académica mediante la creación de nuevas escuelas y modernización de los métodos de enseñanza, g) Asistencia social a los estudiantes, h) Democratización del ingreso a la universidad, i) Vinculación con el sistema educativo nacional, j) Extensión universitaria a través del fortalecimiento de la función social de la Universidad, k) Espíritu democrático por la unidad latinoamericana, 1)Lucha frente a las dictaduras y el imperialismo (López, 2008, p.p. 346/347). 
De todas estas reformas, según reivindica Marsiske (2015) "el distintivo más importante de las universidades latinoamericanas en el siglo XX ha sido el anhelo y después el logro de la autonomía universitaria como precepto legal" (p. 60). Aun cuando se le acercaría en importancia la permanente politización de la universidad pública, en especial sus movimientos estudiantiles involucrados en las luchas de los sectores populares.

Dado el consecuente avance agroexportador de las manufactureras, así como de los servicios destinados al crecimiento del intercambio endógeno, emergieron nuevos sectores sociales medios y populares, los primeros absorbidos por el aparato estatal con su creciente burocracia o a profesiones liberales al servicio de la administración de las empresas exportadoras. Según refiere González (2011) tales colectivos humanos criticaron explícitamente el decadente régimen político surgido de la alianza de los terratenientes y los grupos económicos extranjeros, y se movilizaron por la conquista de participación en el sistema político. "En esas condiciones surgió el discurso positivista articulándose con el movimiento democrático liberal con nacientes corrientes socialistas" (González, 2011).

Situaciones más o menos similares se presentaron en gran parte de América Latina, convirtiéndose en un escenario de enfrentamiento entre las potencias hegemónicas previas a las guerras mundiales, y que luego de concluida esa confrontación inter imperial de reparto del mundo como lo fue la segunda guerra mundial, dejó a Latinoamérica plenamente bajo la órbita de dominación estadounidense, convertido este país en la primera superpotencia atómica.

Las universidades, posteriormente a la institucionalización de la reforma de Córdova, se sintonizaron con las exigencias modernizadoras asumidas por las clases dominantes del subcontinente, poniéndose a disposición de la formación técnica e ideológica de los cuadros medios gerenciales necesarios para el desarrollo de la planta industrial ensambladora que se nos impuso en la nueva división del trabajo, como modelo de inserción en el mercado capitalista, conocido como modelo de sustitución de importaciones. Modelo este que entró en auge en prácticamente toda América Latina (con sus diferencias de tamaño y velocidad) a partir de los años 50 del siglo XX (Bitar y Troncoso, 1983).

La década de los años 60, con el influjo de la revolución cubana, del mayo francés, el movimiento Hippie, la guerra del Vietnam, el poder joven, las universidades fueron conmovidas 
por fuertes movimientos estudiantiles, e incluso a menudo profesorales, orientados a profundizar las reformas de la universidad en la consecución de una Universidad para el pueblo (Caruso citado por Marsiske (2015). Habrían sido estos años tiempos de radicalismo político universitario en la búsqueda de superar las reformas por la revolución, impactando el mundo de las ideas con (por ejemplo) la teología de la liberación. "Pero, según Marcelo Caruso, esta radicalización no sólo tuvo una vertiente política, sino cambió también los paradigmas de las ciencias sociales y de las ciencias naturales y los llevó a nuevo brío" (Marsiske, 2015, p. 64).

La rebeldía juvenil y el auge de las ideas y movimientos revolucionarios, muchos de ellos de corte marxista, que llevaron al inicio de la década del 70 al poder político en Chile al primer presidente latinoamericano de inspiración socialista, Salvador Allende. La reacción de las clases políticas latinoamericanas, apoyadas y empujadas por la élite industrial militar estadounidense fue la imposición de dictaduras militares (Chile, Argentina, Bolivia, Uruguay, Paraguay, Brasil) y gobiernos civiles autoritarios y comprometidos con las doctrinas de seguridad y defensa antisubversivas coordinadas desde la Escuela de las Américas (México, Venezuela,Colombia).

La represión política se afincó con especial brutalidad en las universidades, sus profesores y estudiantes, llegando en muchos casos al allanamiento y cierre de las universidades, como ocurrió con la Universidad Central de Venezuela, o masacres como la de Tlatelolco en México.

En el plano económico nos reseñan Martínez \& Reyes (2012, p. 39) que:

Entre 1950 e inicio de 1980, la región latinoamericana experimentó un notable crecimiento económico, que se vio reflejado en el considerable crecimiento de la renta per cápita, por ejemplo, el incremento para Venezuela fue de 60\%, mientras que para Brasil fue de 240\%. En promedio, la renta per cápita latinoamericana creció 5.492 dólares estadounidenses en 1966. En la década de 1960 y 1970, el crecimiento latinoamericano se caracterizó por las facilidades del financiamiento externo y por el continuo aumento de las exportaciones.

En los años 80 y 90, con la entrada en el ciclo neoliberal, se produjo un salto cualitativo en la orientación ideológica de un significativo número de intelectuales universitarios influyentes en la construcción de los modelos teóricos impartidos en las aulas de clase. El fin de la guerra fría, la disolución de la Unión de Repúblicas Socialistas y Soviéticas (URSS), la caída del muro de Berlín, tendió a plenar de desencanto a la intelectualidad de la izquierda universitaria, traducido 
en desmovilización, deserciones y conversiones, lo cual necesariamente impactaría a la universidad. A la par se inauguraba un nuevo orden mundial caracterizado por la imposición del gobierno norteamericano como hegemón mundial.

Como consecuencia, es allí donde se toman la mayor parte de las decisiones que afectan al resto del mundo. Esto ilustra muy bien una de las características de la globalización neoliberal, la excesiva concentración de poder económico y político en manos de pocos actores - y principalmente en un país - y la asimetría de la concentración espacial del mismo en pocos locales (Mengo, 2010).

En consecuencia, se le impone al subcontinente latinoamericano los designios del llamado Consenso de Washington, ante la crítica situación que se le presenta al subcontinente en la llamada "crisis de la deuda externa" que estructuralmente habría sido ocasionada por: "altos déficit públicos, mercados financieros reprimidos, mercados cambiarios artificialmente controlados, mercados de bienes cerrados a la competencia mundial, restricciones a la inversión extranjera, empresas estatales ineficientes, rígidas relaciones laborales y derechos de propiedad mal definidos" ( Martínez \& Reyes, 2012, p. 43).

Según indican estos autores, el origen de las políticas bautizadas con tal nombre se remonta a una conferencia de expertos académicos de las ciencias económicas, reunidos en noviembre de 1989, proponiendo "reformas que en Washington eran necesarias para restablecer el crecimiento económico de América Latina" (Martínez \& Reyes, 2012, p. 43). Es decir que se impuso la razón tecnocrática asociada al poder de los representantes de los llamados organismos multilaterales, quienes establecieron “diez instrumentos de política económica que Williamson presentó como los objetivos prioritarios, y que los Estados latinoamericanos debían tener para salir del bache económico" (ídem). Razón tecnocrática que tendió a posicionarse en la universidad latinoamericana, legitimada por la disciplina con que los gobiernos estuvieron dispuestos a asumir lo que luego se conoció como "recetario del FMI", a cambio de importantes préstamos. Las políticas económicas recomendadas fueron:

a) Disciplina fiscal, b) Reordenación del gasto público (recorte de gasto público con énfasis en la eliminación de subsidios, c) Reforma fiscal: ampliación de recaudación tributaria, d) Liberalización financiera, especialmente de los tipos de interés bancario, e) Tipo de cambio 
competitivo: promoviendo las exportaciones, f) Liberalización del comercio: reformas arancelarias, g) Liberalización de la inversión extranjera directa, h) eliminación de barreras a la inversión de capital transnacional, i) Privatizaciones, de las empresas en propiedad estatal, j) Desregulación, k)Derechos de propiedad, creación de una legalidad que blindara la propiedad privada.

El costo social de la aplicación de las referidas políticas económicas fue sumamente alto, con agudización de las crisis políticas y sociales que llevaron a rebeliones de sectores desposeídos como la ocurrida en Caracas, Venezuela y la terrible experiencia argentina, dado que "afectó directamente a la distribución del ingreso y polarizó aún más la brecha entre ricos y pobres, agudizando el problema de la pobreza en América Latina” (Martínez \& Reyes, 2012, p. 54).

No obstante, la narrativa neoliberal terminó de posicionarse como especie de sentido común en las universidades latinoamericanas, a finales de los 90 y primeros años del siglo XXI, comienza a cambiar el equilibrio de fuerzas entre los gobiernos de la región luego de la llegada al gobierno por la vía electoral de un proyecto político anti neoliberal, primero en Venezuela, luego en Argentina, Brasil, Bolivia, Ecuador, Nicaragua, para luego extenderse a parte de los pequeños estados insulares del Caribe, generándose con el gobierno socialista cubano un movimiento integracionista de políticas alternativas a la globalización neoliberal y a las medidas dictadas por los entes financieros multilaterales. Las primeras reacciones desde la intelectualidad de las universidades latinoamericanas fue el calificar las propuestas alternativas como retrógradas al pensamiento decimonónico, aun cuando en la práctica política logró el retroceso de las propuestas estadounidenses de creación de un área de libre comercio para las Américas(ALCA).

Posteriormente, en países como Argentina, Brasil, Uruguay, Paraguay y Honduras, las fuerzas partidarias del anti neoliberalismo fueron desplazadas de los respectivos gobiernos, fortaleciéndose en la región las posturas vinculadas y asociadas al pensamiento capitalista neoliberal. De manera tal que el panorama regional se presenta como la contraposición de dos proyectos geopolíticos, lo que necesariamente impactará el devenir de la educación superior, tal como lo plantea Albornoz (2006).

Ahora bien, a fines de la segunda década del siglo XXI sigue posicionada en el mundo académico la idea del mundo globalizado como expresión de las nuevas realidades del mundo de hoy, en el 
contexto de la sociedad del conocimiento. Sin embargo, en el último año y medio, producto del resultado electoral en las elecciones presidenciales estadounidenses, se presentan evidencias interpretadas por analistas políticos y económicos como la adopción de políticas anti globalización (Santana, 2017; Ogglietti \& Martín-Carrillo, 2016 y Rubio, 2018, entre otros).

\section{Los retos de la educación superior en América Latina}

Castell (2003) identifica las problemáticas más relevantes actuales del subcontinente, planteadas como crisis multidimensional, con miras a la discusión de las posibles políticas para enfrentarlas.

a) La débil capacidad tecnológica y de competitividad empresarial en la nueva economía global, b) La inestabilidad financiera, c) La exclusión social de una buena parte de la población, d) El deterioro acelerado del medio ambiente, y, e) La creciente distancia entre las instituciones del Estado y la vivencia de la sociedad. (p. 34/35)

Adicionalmente plantea cuatro grandes problemas:

1. La transición al informacionalismo como nuevo modelo de desarrollo, lo que requeriría de una infraestructura tecnológica centrada en la comunicación y la información; así como una profunda reforma educativa a todo nivel que implicaría: formación de formadores, mejoras remunerativas a los docentes, efectiva utilización de enseñanza virtual. "La Universidad es, potencialmente, la principal fuerza productiva en el nuevo modelo de desarrollo" (Castell, 2003, p. 35).

2. La corrupción en todos los niveles de la administración del Estado.

3. Lo obsolescencia administrativa y crisis de legitimidad política de sus Estados. Avanzar en la democratización acercando al Estado al Ciudadano reforzando gobiernos locales.

\section{Reconstrucción de identidades comunicables.}

Ante tal complejidad de las problemáticas se abren al menos dos posiciones extremas, por un lado, una Universidad orientada a la profundización de la democracia participativa y protagónica, construyendo una economía mixta con orientación distributiva en el marco de un Estado social de derecho y de justicia, con acceso a la educación universal pública y de calidad. En contraposición, otra fundada en la competitividad, el esfuerzo individual, el libre juego de oferta 
y demanda, privatización de los servicios incluyendo la educación, ésta última estratificada en función de las capacidades y estatus social de los aspirantes.

Schwartzman $(\mathrm{S} / \mathrm{F})$ propone que se priorice por la superación de los límites al acceso a la educación universitaria, se amplíe la diversificación de modalidades universitarias acordes a las priorizaciones de una u otra de las funciones de la universidad. Priorización de las formas de vinculación con el mercado laboral y la Universidad. Promover mayor abertura y sensibilidad de las instituciones de educación superior hacia las demandas e intereses de la sociedad. Propiciar reformas puntuales y no generales en el sistema educativo. Afinar y propiciar los mecanismos e instrumentos de evaluación institucional de la calidad educativa. Incrementar progresivamente las competencias empresariales y de servicios de las universidades con miras a su mayor autofinanciamiento.

Desde una perspectiva institucional, vinculada con el órgano especializado de las Naciones Unidas para la educación y la cultura (UNESCO- IESALC), se han identificado entre los desafíos actuales de la Universidad en el subcontinente:

1. fortalecer la interface entre universidades de la región para incrementar intercambios académicos y culturales;

2. maximizar estudios sistemáticos sobre teorías y modelos de gestión aplicados a las universidades regionales;

3. profundizar la discusión sobre la vulnerabilidad de las instituciones universitarias de cara a las crisis y transformaciones actuales; $y$

4. repensar las estrategias de desarrollo para la enseñanza, investigación, extensión y gestión de las universidades

\section{Conclusión}

La Universidad latinoamericana ha estado sintonizada y por tanto ha respondido de manera dialéctica frente a los cambios y desafíos que se ha planteado su compleja y plurinacional y multicultural sociedad. Al menos desde la reforma de Córdova, con sus dinámicas políticas derivadas de los movimientos estudiantiles y gremiales ha procurado adaptarse a las demandas 
provenientes de los cambios sociales, con una perspectiva modernizadora. Aun cuando no siempre se pone al servicio de las clases populares, es su tendencia prioritaria. Los vigentes cambios políticos, económicos y sociales le plantean retos que al menos pueden ser orientados en dos perspectivas que se encuentran en tensión en nuestros países: Socialismo vs neoliberalismo, aun cuando no se descarta que surjan salidas más eclécticas para la necesaria transformación universitaria en función de sus necesidades más sentidas.

\section{Referencias Bibliográficas}

Albornoz, O. (2006) La Universidad latinoamericana entre Davos y Porto Alegre: error de origen, error de proceso. Venezuela: Editorial CEC, SA

Bitar, S. \& Troncoso, E. (1983) El desafío industrial de Venezuela. Argentina: Pomaire.

Castells, M. (2003) Panorama de la era de la información en América Latina: ¿es sostenible la globalización? En Calderón,F (Coord). ¿es sostenible la globalizaciónen América Latina? Debates con Manuel Castells, volumen II. Nación y cultura. América Latina en la era de la información. México: Fondo de Cultura Económica/ PNUD Bolivia.

González, R. (10 de octubre de 2011) La reforma universitaria argentina de 1918. (Mensaje en un Blog) recuperado de: http://paginalatina.blogspot.com/2011/10/la-reforma-universitariaargentina-de.html

IESALC (20 de noviembre de 2017) La Sociedad del Conocimiento será el tema central del XVII Coloquio Internacional de Gestión Universitaria (CIGU) en Mar de Plata, Argentina. Recuperado de: http://www.iesalc.unesco.org.ve/index.php?option=com_content\&view=article\&id=3937:1asociedad-del-conocimiento-sera-el-tema-central-del-xvii-coloquio-internacional-de-gestionuniversitaria-cigu-en-mar-de-plata-argentina\&catid=11\&Itemid=466\&lang=es

López, A. (2008). Ausencia de las reformas de Córdoba en las universidades de Venezuela (1918-1935). Educere, 12(41), 337-350. Recuperado de:

Marsiske, R. (2015) La universidad latinoamericana en el siglo XX: una aproximación. Universidades [en linea], (Julio-Septiembre): Recuperado de: http://www.redalyc.org/articulo.oa?id=37341213006 
Martínez R., R., \& Reyes G., E.. (2012). El Consenso de Washington: la instauración de las políticas neoliberales en América Latina. Política y cultura, №37, 35-64. Recuperado de http://www.scielo.org.mx/scielo.php?script=sci_arttext\&pid=S0188-

$77422012000100003 \& \operatorname{lng}=$ es\&tlng=es

Mengo, R. (2010). Desde la caída del Muro de Berlín a la Crisis económica Internacional Cambios y sucesos en el Sistema Mundial- Revista Globalización. Recuperado de: http://rcci.net/globalizacion/2010/fg968.htm

Ogglietti, G. Martín-Carrillo, S. (11 de noviembre de 2016) Por qué ganó Trump y la crisis del modelo de globalización actual. Telesur. Recuperado de: https://www.telesurtv.net/opinion/Porque-gano-Trump-y-la-crisis-del-modelo-de-globalizacion-actual-20161111-0011.html

Rubio, F. (26 de enero de 2018) La globalización interrumpida por Donald Trump. Disidentia. Recuperado de: https://disidentia.com/globalizacion-interrumpida-donald-trump/

Saber ULA (S/F) La Reforma Universitaria de Córdova de 1918, recuperado de: www.saber.ula.ve/bitstream/123456789/22014/1/articulo2.pdf

Santana, R, (01 de febrero de 2017). Trump: la globalización y la hegemonía norteamericana. Listín Diario. Recuperado de: https://www.listindiario.com/economia/2017/02/01/452481/trumpla-globalizacion-y-la-hegemonia-norteamericana

Schwartzman, S. (S/F) Las Universidades Latinoamericanas en Contexto. recuperado de: www.ufg.edu.sv/ufg/theorethikos/Julio02/universi.htm 